\title{
Experimental tests on operation performance of a LARM leg mechanism with 3-DOF parallel architecture
}

\author{
M. F. Wang, M. Ceccarelli, and G. Carbone \\ LARM: Laboratory of Robotics and Mechatronics, DICeM-University of Cassino and South Latium, Cassino \\ (Fr), Italy \\ Correspondence to: M. F. Wang (wang@unicas.it)
}

Received: 9 July 2014 - Revised: 17 December 2014 - Accepted: 13 January 2015 - Published: 30 January 2015

\begin{abstract}
In this paper, a prototype of a LARM leg mechanism is proposed by using a tripod manipulator and its operation performance is investigated through lab experimental tests. In particular, an experimental layout is presented for investigating operational performance. A prescribed motion with an isosceles trapezoid trajectory is used for characterizing the system behavior. Experiment results are analyzed for the purpose of operation evaluation and architecture design characterization of the tripod manipulator and its proposed prototype.
\end{abstract}

\section{Introduction}

Legged locomotion has a number of advantages as compared with conventional wheeled and crawler-type locomotion, such as higher mobility, better obstacle overcoming ability, energy efficiency, active suspension and achievable speed, especially when it operates in rough or unconstructed environment (Pfeiffer, 2004; Carbone and Ceccarelli, 2005; Siciliano and Khatib, 2008).

Biped locomotors, as a significant hot topic, have attracted interests of many research communities in the past decades, and a lot of prototypes have been built in the laboratories and even for specific application tasks (Carbone and Ceccarelli, 2005; Siciliano and Khatib, 2008). In addition, parallel manipulators are well known for higher payload capability, stiffness, accuracy and dynamic performance in contrast to traditional serial manipulators, and have been widely studied both in industry and academia (Ceccarelli, 2004; Merlet, 2006). However, most of the existing biped locomotors are based on leg designs with human-like architectures by using serial chain solutions, such as ASIMO (Sakagami et al., 2002), HUBO (Park et al., 2005), HRP (Kaneko et al., 2002) and so on. WL-16 (Waseda Leg-No.16) is a design that achieved world first dynamic biped walking as based on leg designs with Gough-Stewart parallel manipulators (Hashimoto et al., 2009). Ota et al. (1998) and Sugahara et al. (2002) have also proposed to use Gough-Stewart parallel manipulators for leg modules in other systems. Neverthe- less, the potentiality of parallel manipulators for leg mechanisms has not been fully investigated, since the typical six degrees of freedom (DOFs) manipulators also suffer from some disadvantages, e.g., limited workspace, difficult mechanical design, complex direct kinematics, and control algorithms. To overcome the above disadvantages, parallel manipulators with fewer than six DOFs, namely reduced DOF manipulators, have been widely studied both in industry and academia (Tsai, 1999; Merlet, 2006). In the field of leg designs for biped locomotors, Ceccarelli and Carbone (2009) have investigated the possibility of using parallel manipulator mechanisms with less than six DOFs for leg designs as inspired from the human leg muscular system. It is worthy to note that several architectures of 3-DOF purely translational parallel mechanisms (TPMs) like Delta in Clavel (1988), Orthoglide in Chablat and Wenger (2003), or others like those in Tsai and Joshi (2001) can be used for leg designs, since turning capability of the biped locomotors can be solved by the waist rotation.

In general, the cost features can be related to the mechanical design mainly concerning with joints and actuators, and to the control equipment both in software and hardware, with numerical evaluations that according to authors' knowledge are not reported in the literature. Hence, from the viewpoints of low-cost and easy-operation, although both TPMs in Clavel (1988) and Chablat and Wenger (2003) show regular workspace and proper dynamic performance, the rela- 


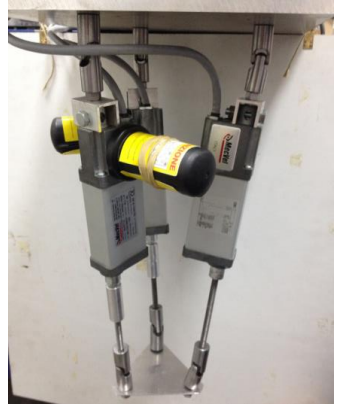

(a)

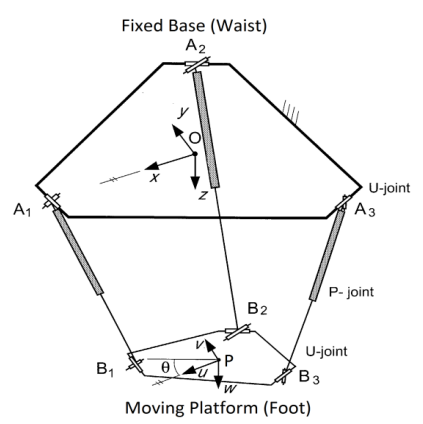

(b)
Figure 1. A design of LARM tripod leg mechanism: (a) a prototype at LARM; (b) a kinematic scheme of 3-UPU parallel manipulator.

tively large required operation space and high cost of parallelogram pairs containing S- or U-joints (where S and U stand for spherical and universal pairs, respectively) cannot be considered suitable for leg designs in biped locomotors. In addition, by comparing the four 3-DOF TMPs in the Tsai and Joshi (2001), the rail guides of linear actuators in 3PUU TMPs (where $\mathrm{P}$ stands for a prismatic pair) make the fixed platform too large to be a waist for biped locomotors, while 3-RUU and UPU TMPs (where R stands for a revolute pair) can be expected to be useful for leg designs. Furthermore, Bhutani and Dwarakanath (2014) have presented a high-precision prototype as based on 3-UPU TMP and they validated the practical feasibility of this design in terms of repeatability and trajectory following accuracy for various payloads.

Although few existing prototypes of biped locomotors have been built as based on leg designs with 3-DOF TMPs, there are some other legged locomotors with 3-DOF leg mechanisms. Zhang and Li (2011) have presented a walking locomotor as based on a 3-RPC parallel mechanism by a specific operation mode with two moving platforms. Wang et al. (2009) have proposed a quadruped/biped reconfigurable walking locomotor as based on four 3-UPU parallel leg mechanisms for quadruped walking and by converting them into two 6-SPU parallel mechanisms for biped working. Pan and Gao (2013) have presented a hexapod walking locomotor for situations when a nuclear disaster happens and it is based on 3-DOF parallel mechanisms. In addition, parallel manipulators with 3-DOF, have been widely investigated for relevant applications and they have simpler structure and kinematics, larger workspace, and more convenient control with respect to hexapods (Merlet, 2006). Indeed, 3-DOF manipulators could be enough for reducing the total cost and operations of leg mechanisms for biped locomotors.

At the Laboratory of Robotics and Mechatronics (LARM) of University of Cassino and South Latium, a research line is devoted to design and analysis of parallel mechanisms with reduced DOFs for multiple purposes, and several prototypes of parallel manipulators have been built with low-cost and

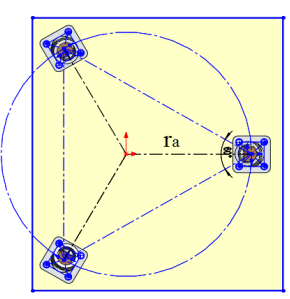

(a)

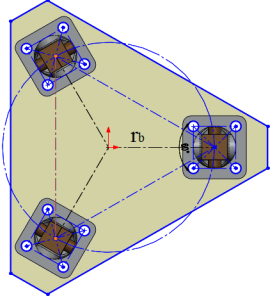

(b)
Figure 2. A scheme of arrangement of U-joints: (a) in the upper plate (waist); (b) in the lower plate (foot).
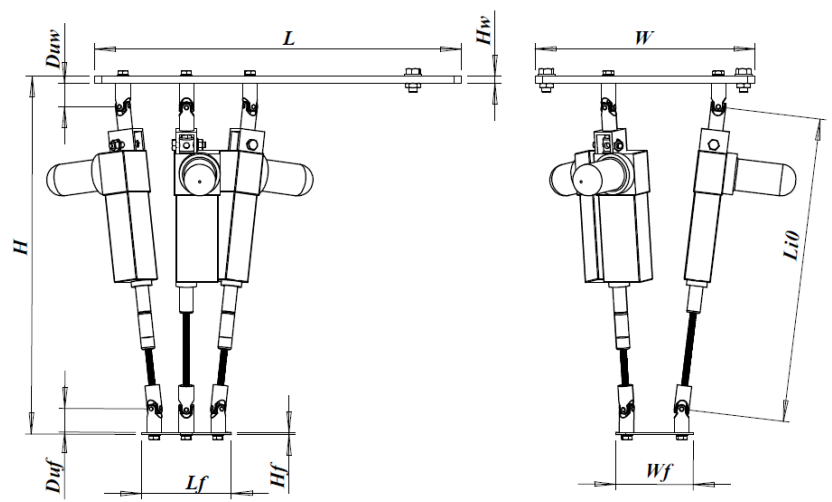

Figure 3. Dimension parameters of the prototype in Fig. 1a.

easy-operation characteristics (Ceccarelli, 2012). Research activities have been carried out on both theoretical study and application aspects. A 3-DOF parallel manipulator has been proposed, studied and used as a novel design solution of a LARM tripod leg mechanism for a biped locomotor (Wang and Ceccarelli, 2013).

In this paper, a prototype of a LARM leg mechanism is presented as based on a 3-DOF parallel manipulator. The experimental layout of the foot motion control system is presented for experimental experiences. Operational performance of the proposed LARM tripod leg mechanism has been investigated by experimental tests. A fairly simple motion with an isosceles trapezoid trajectory is prescribed for characterizing the system behavior. Experimental results are analyzed for the purpose of operation performance evaluation and architecture design characterization of the proposed LARM tripod leg mechanism.

\section{A LARM tripod leg mechanism}

A prototype of the proposed LARM tripod leg mechanism and its kinematic scheme of a 3-UPU parallel manipulator are shown in Fig. 1a and b, respectively. The tripod leg mechanism is a parallel manipulator consisting of a waist plate, a moving foot plate, and three identical limbs of linear actuators with U-joints at each end. Hence, in the tripod leg 
Table 1. Main specifications of LARM tripod leg mechanism

\begin{tabular}{lcccc}
\hline DOF & Weight $(\mathrm{kg})$ & Dimension $(L \times W \times H \mathrm{~mm})$ & Step size $\left(S_{\mathrm{L}} \times S_{\mathrm{H}} \mathrm{mm}\right)$ & Step cycle (s/step) \\
\hline 3 & 4.5 & $223 \times 200 \times 463$ & $200 \times 50$ & 6 \\
\hline
\end{tabular}

mechanism, there are eight links that are connected by six Ujoints and three P-joints, and the DOFs of the mechanism can be calculated as 3 from the expression of Grübler-Kutzbach criteria

$F=6(n-j-1)+\sum_{i=1}^{j} f_{i}$

Since the joint DOFs of each limb are equal to five, each limb provides one constraint to the moving platform. As shown in Fig. 1b, U-joints in each limb are arranged with two outer revolute joint axes that are parallel to each other and the two inner revolute joint axes that are parallel to one another, so that each limb provides one rotational constraint to the moving platform. A combination of three limbs in each leg completely constrains the moving platform from any instantaneous rotation. Hence, since the limb constraints are independent from each other, the moving platform possesses purely translational motion, as indicated in (Tsai and Joshi, 2000).

Additionally, the upper and lower three U-joints are installed in equilateral triangle arrangement with one ahead and the other two rear, as shown in Fig. 2, where each three inner revolute axes are installed pointing to the corresponding circumcenter of the triangle and the circumradiuses are $r_{\mathrm{a}}$ and $r_{\mathrm{b}}$, respectively.

The main specifications and details of mechanical design parameters of the LARM tripod leg mechanism are listed in Tables 1 and 2, as referring to dimension parameters in Fig. 3, where $L, W$, and $H$ are length, width and height of the mechanism, respectively; $S_{\mathrm{L}}$ and $S_{\mathrm{H}}$ are step length and height of the foot; $H_{\mathrm{w}}$ and $H_{\mathrm{f}}$ are the thicknesses of waist and foot plates; $L_{\mathrm{f}}$ and $W_{\mathrm{f}}$ are length and width of the foot, respectively. In each limb, the distance between the rotation center of upper U-joint and waist plate is equal to the distance between the rotation center of lower U-joint and foot plate, i.e. the half length of the universal joint, given by $D_{\mathrm{uw}}=D_{\mathrm{uf}}$. The distance between the two rotation centers of U-joints, is the length of each limb that is indicated as $l_{i}(i=1,2,3)$, with the initial value given as $L_{i 0}$, which determines the initial height of the proposed mechanism. Finally, the stroke of linear actuators is indicated as $L_{\mathrm{s}}$.

Since the LARM tripod leg mechanism is developed for biped locomotors, which will be capable of moving with flexibility and versatility in practical applications, during the activity for mechanical design, particular attention has been paid to characteristics for low cost solution, load capacity, easy operation, lightweight and compact design. The proposed solution is worked out by choosing proper commercial
Table 2. Mechanical design parameters of LARM tripod leg mech$\operatorname{anism}(\mathrm{mm})$.

\begin{tabular}{ccccccccc}
\hline$H_{\mathrm{w}}$ & $H_{\mathrm{f}}$ & $L_{\mathrm{f}}$ & $W_{\mathrm{f}}$ & $r_{\mathrm{a}}$ & $r_{\mathrm{b}}$ & $D_{\mathrm{uw}}=D_{\mathrm{uf}}$ & $L_{i 0}$ & $L_{\mathrm{s}}$ \\
\hline 10 & 3 & 122.4 & 106 & 100 & 50 & 25 & 403 & 100 \\
\hline
\end{tabular}

products, which have been also used in design modelling, and by adopting aluminium alloy as the material for the plates of waist and foot for its stiffness, mass density, and cheap cost.

For kinematic analysis, a static coordinate frame A: O-xyz and a moving coordinate frame B: P-uvw are assumed on the fixed base and moving platforms, and points $O$ and $P$ are the centers of platforms, respectively, as shown in Fig. 1b. A position vector $\boldsymbol{p}=\left[p_{x}, p_{y}, p_{z}\right]^{T}$ of a reference point $P$ in the center of moving platform is given for indexing walking performance. The $i$ th $(i=1, \ldots, 3)$ actuated limb is connected to the moving platform at point $B_{i}$ and to the fixed base at point $A_{i}$, where $A_{i}$ and $B_{i}$ are the rotation center of corresponding U-joint. Furthermore, points $A_{i}(i=1, \ldots, 3)$ lie on an equilateral triangle in the $O-x y$ plane at a radial distance of $r_{\mathrm{a}}$ from point $O$, and $B_{i}(i=1, \ldots, 3)$ lie on an equilateral triangle in the P-uv plane at a radial distance of $r_{\mathrm{b}}$ from point $P$. Hence, the position of the moving platform and length of each limb can be respectively obtained in closed-form through the expressions as

$$
\begin{aligned}
& p_{x}=\left(-2 l_{1}^{2}+l_{2}^{2}+l_{3}^{2}\right) / 6 w \\
& p_{y}=\left(l_{3}^{2}-l_{2}^{2}\right) / 2 \sqrt{3} w \\
& p_{z}=\sqrt{l_{1}^{2}-\left(p_{x}-w\right)^{2}-p_{y}^{2}} \\
& l_{1}=\sqrt{\left(p_{x}-w\right)^{2}+p_{y}^{2}+p_{z}^{2}} \\
& l_{2}=\sqrt{\left(p_{x}+w / 2\right)^{2}+\left(p_{y}-\sqrt{3} w / 2\right)^{2}+p_{z}^{2}} \\
& l_{3}=\sqrt{\left(p_{x}+w / 2\right)^{2}+\left(p_{y}+\sqrt{3} w / 2\right)^{2}+p_{z}^{2}}
\end{aligned}
$$

where $w=r_{\mathrm{a}}-r_{\mathrm{b}}$, and the negative values for limb length and root of $p_{z}$ cannot be considered for this mechanism. Hence, the displacement $L_{i}$ of each linear actuator can be expressed as

$$
L_{i}=l_{i}-L_{i 0}
$$




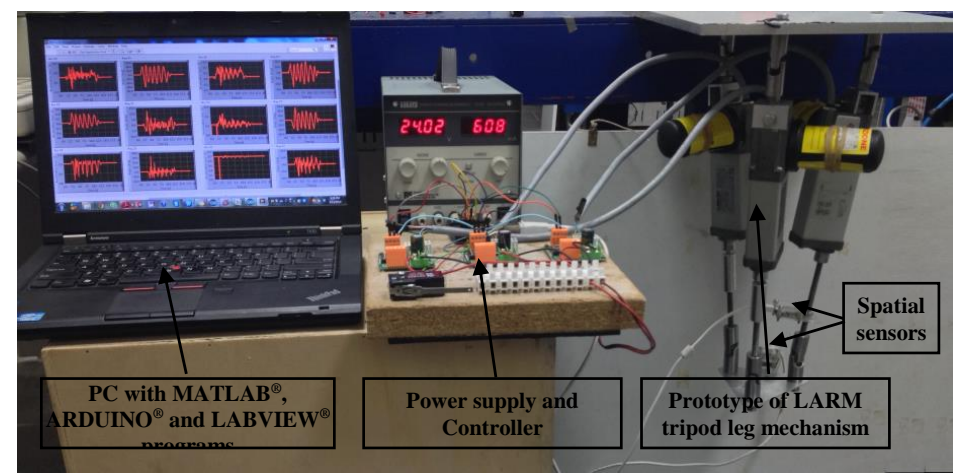

(a)

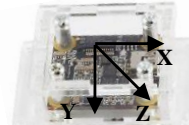

(b)

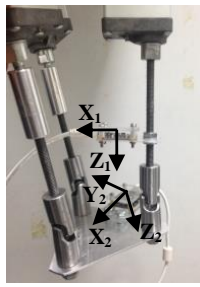

(c)

Figure 4. Layout for a foot motion control system of the proposed LARM tripod leg mechanism for experimental tests: (a) a layout of whole system; (b) a Phidgets ${ }^{\circledR}$ spatial 3/3/3 1044_0 sensor; (c) Location of two sensors.

Part I

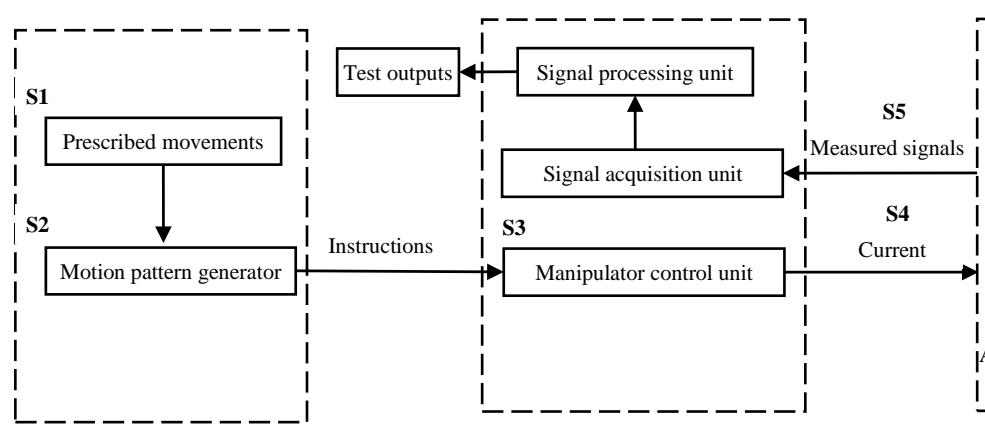

Part III

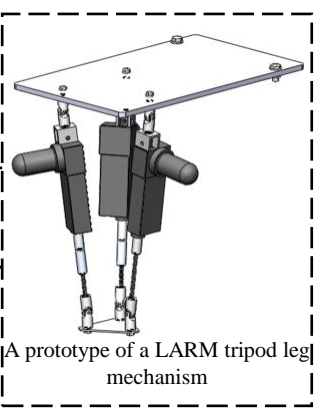

Figure 5. A schematic diagram of the experimental layout in Fig. 4 for an operation procedure.

\section{An experimental layout and test modes}

Figure 4 shows the foot motion control system of the proposed prototype of the LARM tripod leg mechanism for experimental tests. In Fig. 4a, the leg mechanism is actuated by three linear actuators with $24 \mathrm{~V}$ DC motors which are controlled by three AQMD2410NS DC motor drive cards with instructions from an ARDUINO card. Figure $4 b$ shows a spatial Phidgets ${ }^{\circledR}$ sensor which has been used to measure acceleration, angular rate, and magnetic field strength in the Cartesian space. In Fig. 4c, two similar spatial sensors that are fixed on the linear actuator and the foot platform, respectively, are used for measuring the acceleration of linear actuators along $z$ axis and the angle information of the foot platform along three axes.

Figure 5 shows a scheme of the experimental operation procedure for the prototype of LARM tripod leg mechanism during experimental tests. The whole system consists of three parts: Part I is a motion pattern generator with suitable program running in MATLAB ${ }^{\circledR}$ environment; Part II consists of the manipulator control unit, signal acquisition unit, and PC with LABVIEW ${ }^{\circledR}$ software; Part III is the built prototype of the LARM tripod leg mechanism.
A controlled operation can be performed by following five steps (S1 to S5), which can be described as

- S1: it gives inputs of the motion generator for the leg mechanism of the prototype in Fig. 1a;

- S2: displacements for the three linear actuators are computed by using Eqs. (3) and (4) in MATLAB ${ }^{\circledR}$ environment;

- S3: the computed motion trajectories in the motion pattern generator are transformed to control instructions in ARDUINO $^{\circledR}$ environment, as the inputs of the manipulator controllers;

- S4: each limb follows the prescribed motion trajectory by driving DC motor of linear actuator under an openloop control;

- S5: for each period of sampling time, angle positions of the moving platform and accelerations of each linear actuator are measured in LABVIEW ${ }^{\circledR}$ environment, and they are stored in the PC for data analysis and characterization purposes of operation performance. 
Table 3. Prescribed motion parameters of LARM tripod leg mechanism (mm).

\begin{tabular}{lrrrrrrr}
\hline Axis & $P_{0}(t=0 \mathrm{~s})$ & $P_{1}(t=1 \mathrm{~s})$ & $P_{2}(t=2 \mathrm{~s})$ & $P_{3}(t=3 \mathrm{~s})$ & $P_{4}(t=4 \mathrm{~s})$ & $P_{5}(t=5 \mathrm{~s})$ & $P_{6}(t=6 \mathrm{~s})$ \\
\hline$x$ & 0 & 50 & 100 & 0 & -100 & -50 & 0 \\
$y$ & 0 & 0 & 0 & 0 & 0 & 0 & 0 \\
$z$ & 400 & 400 & 450 & 450 & 450 & 400 & 400 \\
\hline
\end{tabular}

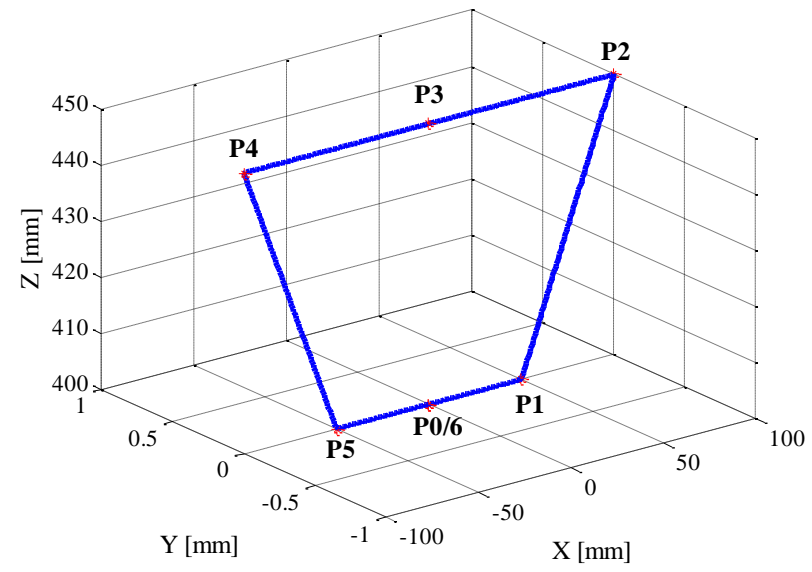

Figure 6. A prescribed input motion for experimental test.

\section{Experimental test results}

For human normal walking, the motion of a leg can be divided into two phases, i.e. a swing phase and a supporting phase (Carbone and Ceccarelli, 2005), and the human foot can be considered as the end-effector for a leg since it is moved to achieve proper motions and actions during the leg movements.

In general, the trajectory of a human-liked foot step is an ovoid curve where the straight line segment represents the supporting phase and the curve segment represents the swinging phase (Rose and Gamble, 2006). In this section, a prescribed input motion of foot platform has been considered for experimental experiences, as shown in Fig. 6, where the curve segment is simplified by linear segments in $\mathrm{O}-\mathrm{XZ}$ plane (Zielinska, 2004). Each step of the motion, whose trajectory actually is an isosceles trapezoid, is divided into six segments that are identified by seven prescribed positions, as shown in Table 3, where the start and end values $P_{0}$ and $P_{6}$ are equally given as the initial position of the foot motion. Since the three linear actuators in the prototype are driven under a position and velocity open-loop control, the prescribed displacements are computed in MATLAB ${ }^{\circledR}$ environment and then they are transformed into ARDUINO ${ }^{\circledR}$ programs. The displacements of three linear actuators can be obtained accordingly as shown in Fig. 7, where L2 and L3 are coincident because of the structure symmetry and no offset of the foot in $y$ axis, which can be easily obtained from Eq. (3).

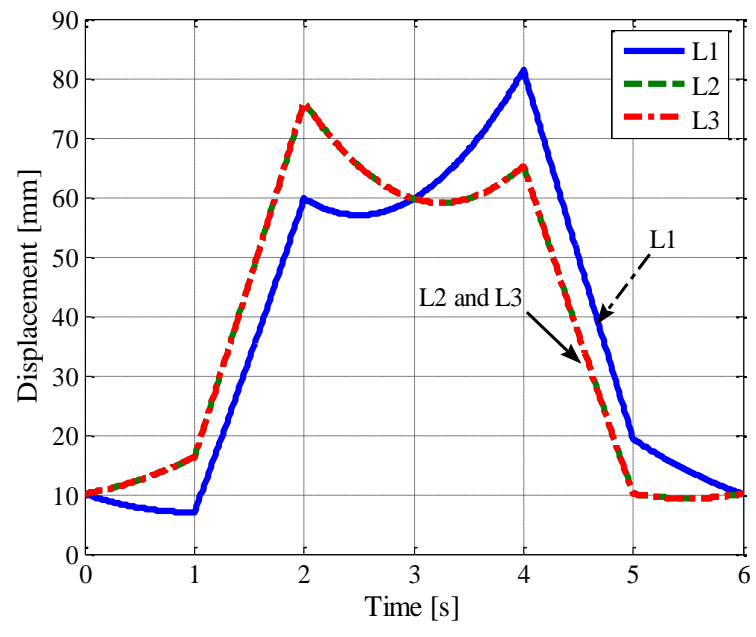

Figure 7. Input displacements of the three linear actuators.

Figure 8 shows the measured rotation angles of the foot platform about three axes. It takes $6 \mathrm{~s}$ for the tested prototype to finish a prescribed movement. The foot platform rotates from -4.0 to $4.5^{\circ}$ about $x_{2}$ axis and from -3.2 to $3.2^{\circ}$ about $z_{2}$ axis, while it rotates from -14.1 to $10.9^{\circ}$ about $y_{2}$ axis. In addition, the rotation angles about $y_{2}$ axis are normally between from -7.5 to $3^{\circ}$, and the two peak values only happen at about $t=2.2 \mathrm{~s}$ and $t=4.1 \mathrm{~s}$, i.e., close to points $P_{2}$ and $P_{4}$. From the test results with micro rotation angles of the foot platform, it can be considered that the foot platform always maintains relatively translational motion but with certain pitch at the extreme positions as points $P_{2}$ and $P_{4}$.

Figure 9 shows the measured linear accelerations of the three linear actuators. According to the prescribed input motions of the three linear actuators, as shown in Fig. 7, the motions of L2 and L3 should be the same, so that only linear accelerations of L1 and L2 are measured and plotted. Since the prescribed velocity of linear actuators in each segment is approximately constant, the accelerations should be approximately equal to zero, but at the beginning of each segment, i.e. at points $\mathrm{Pi}(i=0, \ldots, 5)$ in Fig. 6 , a sudden variation of acceleration occurs because of the velocity changes, which can be deduced by the input motion in Fig. 7. In addition, maximum and minimum acceleration values of $\mathrm{L} 1$ and $\mathrm{L} 2$ are measured as $A_{\mathrm{L} 1 \max }=$ $0.96 \mathrm{~m} \mathrm{~s}^{-2}, A_{\mathrm{L} 1 \min }=-1.34 \mathrm{~m} \mathrm{~s}^{-2}$ and $A_{\mathrm{L} 2 \max }=0.98 \mathrm{~m} \mathrm{~s}^{-2}$, $A_{\mathrm{L} 2 \mathrm{~min}}=-1.28 \mathrm{~m} \mathrm{~s}^{-2}$, respectively. 


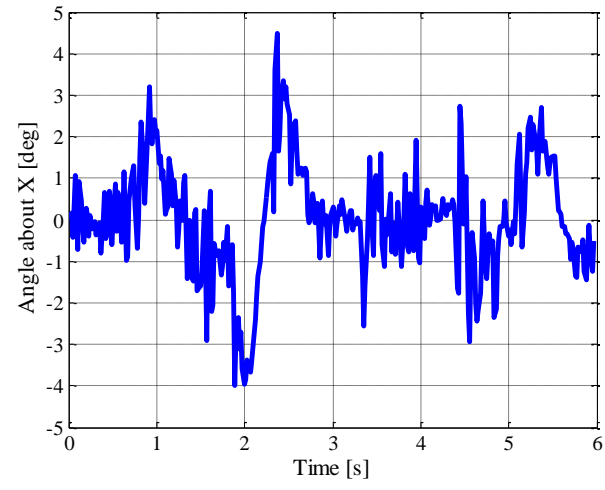

(a)

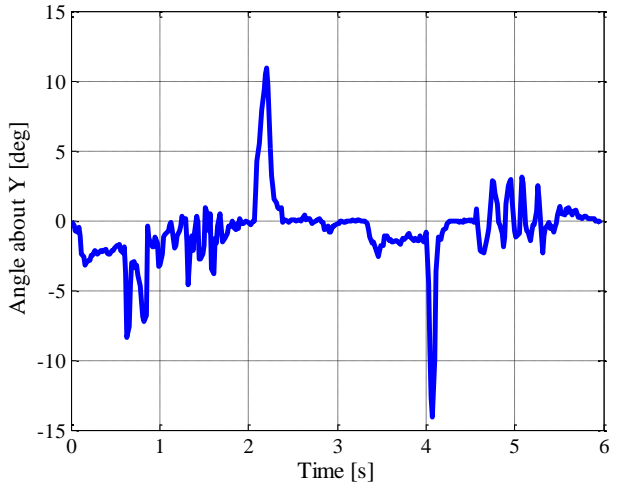

(b)

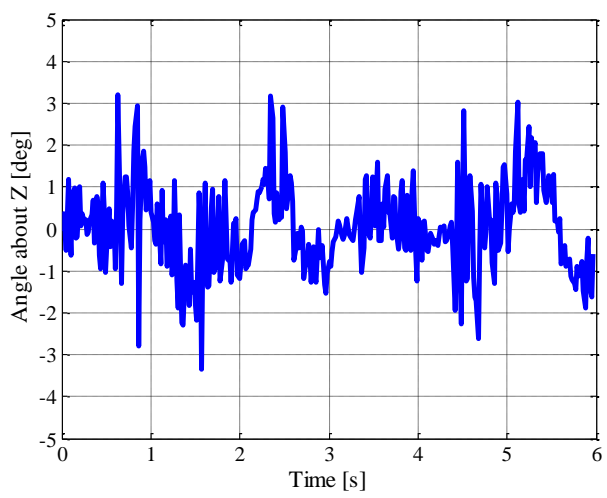

(c)

Figure 8. Measured rotation angles of the foot platform: (a) about $x_{2}$ axis; (b) about $y_{2}$ axis; (c) about $z_{2}$ axis.

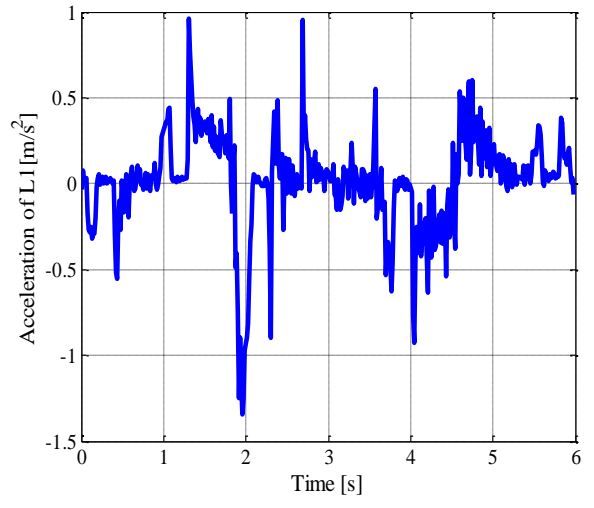

(a)

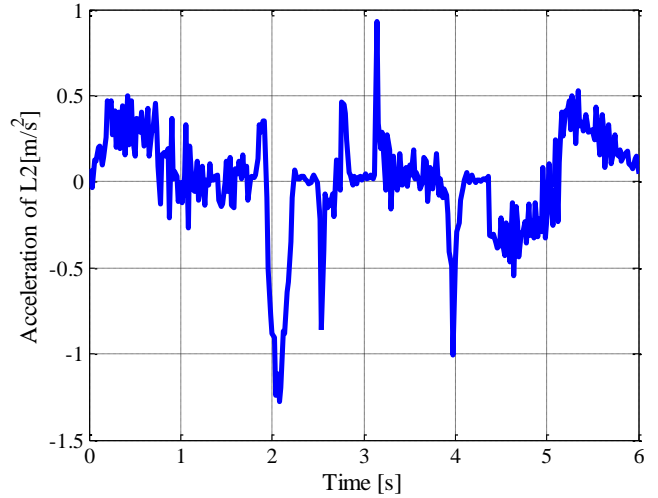

(b)

Figure 9. Measured axial linear acceleration along $z$ axis for two linear actuators: (a) actuator L1; (b) actuator L2.

Figure 10 shows a sequence of leg configurations during an experimental test. Experimental test time has been indicated for each snapshot, which is coincident with the prescribed motion parameters in Table 3. It can be noted that the prototype starts from $\mathrm{P} 0$ and follows the prescribed trajec- tory by six reference points, which are marked by red labels in Fig. 10.

From the experimental test results, it can be noted that the built prototype of the LARM tripod leg mechanism in Fig. 4 can perform a predefined movement with the aim to follow an isosceles trapezoid trajectory. Since it has been experienced 

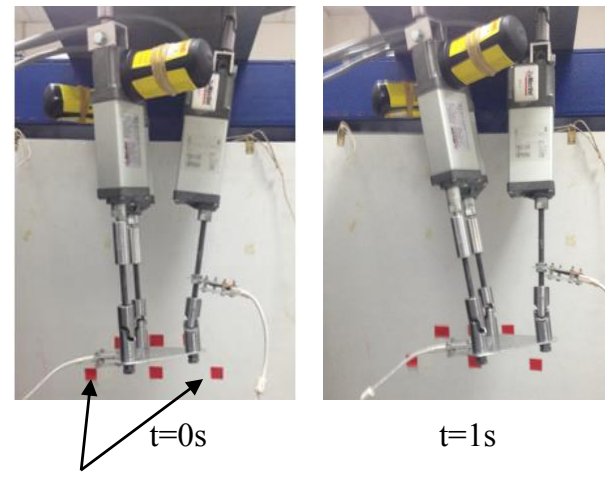

$\mathrm{t}=1 \mathrm{~s}$

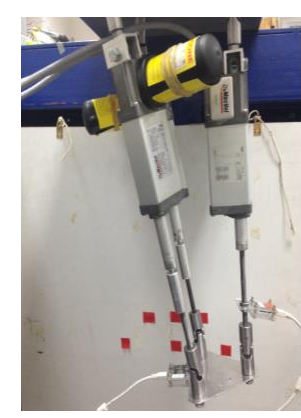

$\mathrm{t}=2 \mathrm{~s}$

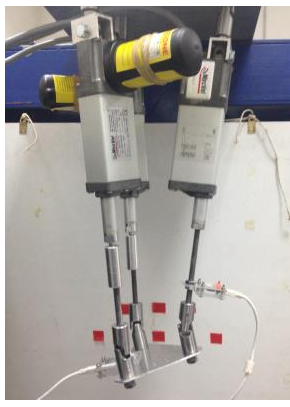

$\mathrm{t}=3 \mathrm{~s}$

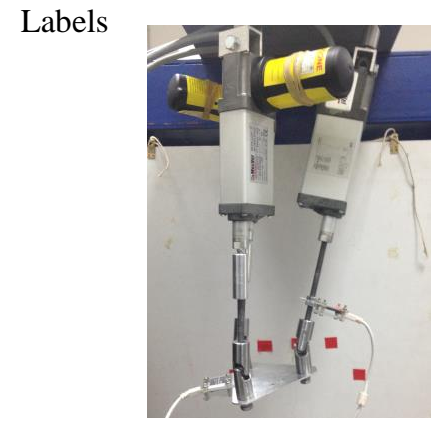

$\mathrm{t}=4 \mathrm{~s}$

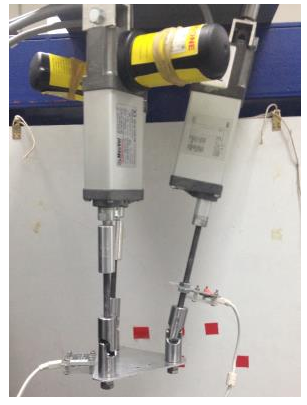

$\mathrm{t}=5 \mathrm{~s}$

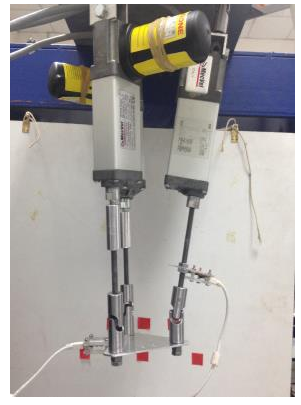

$\mathrm{t}=6 \mathrm{~s}$

Figure 10. A sequence of snapshots of the prototype during a test following the prescribed motion for the test.

difficultly to maintain the relationship between U-joints in each limb during the operation, the motion of the foot platform has shown purely translational movements but with certain pitch at the extreme positions for points $\mathrm{P} 2$ and $\mathrm{P} 4$ in Fig. 6. In addition, the precision of the proposed tripod leg mechanism is function of errors in mechanical design and control equipment. In particular, each of the used linear actuator consists of a $24 \mathrm{~V}$ permanent magnet motor that is coupled to an ACME lead screw whose lead is $2 \mathrm{~mm}$ and actuation error can be estimated in $0.2 \mathrm{~mm}$. The motor drive card is based on current control whose resolution is $0.1 \mathrm{~A}$ and estimated error is about $0.02 \mathrm{~A}$. By considering the stroke and current range of the linear actuators, which are $100 \mathrm{~mm}$ and 1 to $7 \mathrm{~A}$, respectively, the proposed arrangement provides relatively low control resolution along the prismatic motion to the limbs of the tripod leg mechanism. Nevertheless, since the tripod leg mechanism is built for a biped locomotor, small errors of rotation angles of the foot platform can be considered acceptable during the practical application also because they give an operation that is comparable with human walking. The experimental test results are also quite useful for identifying the operation performance of the prototype, validating the mechanical design, and looking for enhancements.

\section{Conclusions}

A prototype of a LARM tripod leg mechanism has been built by using a 3-DOF parallel manipulator. The built prototype is a mechanical design solution with low-cost easy-operation features that can be useful for a tripod leg mechanism in a biped locomotor. Experimental tests have been performed successfully with the prototype to follow a prescribed step movement of the foot platform. Test results have validated the feasibility of the proposed design and have characterized its operation with suitable motion characteristics as a tripod leg mechanism for biped locomotors.

Acknowledgements. The first author would like to acknowledge China Scholarship Council (CSC) for supporting his PhD study and research at the Laboratory of Robotics and Mechatronics (LARM) in the University of Cassino and South Latium, Italy, for the years 2013-2015.

Edited by: A. Barari

Reviewed by: V. Glazunov and one anonymous referee 


\section{References}

Bhutani, G. and Dwarakanath, T. A.: Practical Feasibility of A High Precision 3-UPU Parallel Mechanism, Robotica, 32, 341-353, 2014.

Carbone, G. and Ceccarelli, M.: Legged Robotic Systems, Cutting Edge Robotics, ARS Scientific Book, Vienna, 553-576, 2005.

Ceccarelli, M.: Fundamentals of Mechanics of Robotic Manipulation, Kluwer Academic Publishers, Dordrecht, 2004.

Ceccarelli, M.: An Illustrated History of LARM in Cassino, in: Proc. of Int. Workshop on Robotics in Alpe-Adria-Danube Region (RAAD), Naples, Edizioni Scientifiche e Artistiche, 85-92, 2012.

Ceccarelli, M. and Carbone, G.: A New Leg Design with Parallel Mechanism Architecture, in: Proc. of Int. Conf. on Advanced Intelligent Mechatronics (AIM), Singapore, 1447-1452, 2009.

Chablat, D. and Wenger, P.: Architecture Optimization of A 3-DOF Translational Parallel Mechanism for Machining Applications, the Orthoglide, IEEE Trans. Robot. Autom., 19, 403-410, 2003.

Clavel, R.: Delta, A Fast Robot with Parallel Geometry, in: Proc. of the 18th Int. Symposium on Industrial Robots, Lausanne, 91$100,1988$.

Hashimoto, K., Sugahara, Y., Lim, H. O., and Takanishi, A.: Biped Landing Pattern Modification Method and Walking Experiments in Outdoor Environment, J. Rob. Mechat., 20, 775-784, 2009.

Kaneko, K., Kanehiro, F., Kajita, S., Yokoyama, K., Akachi, K., Kawasaki, T., and Isozumi, T.: Design of Prototype Humanoid Robotics Platform for HRP, in: Proc. of IEEE/RSJ Int. Conf. on Intelligent Robots and Systems (IROS), Lausanne, 3, 24312436, 2002.

Merlet, J. P.: Parallel Robots (2nd Edn.), Springer, Dordrecht, 2006.

Ota, Y., Inagaki, Y., Yoneda, K., and Hirose, S.: Research on a SixLegged Walking Robot with Parallel Mechanism, in: Proc. of Int. Conf. on Intelligent Robots and Systems (IROS), Victoria, 241248, 1998.

Pan, Y., and Gao, F.: Payload Capability Analysis of A New Kind of Parallel Leg Hexapod Walking Robot, in: Proc. of Intl. Conf. on Advanced Mechatronic Systems (ICAMechS), Luoyang, 541544, 2013.

Park, I. W., Kim, J. Y., Lee, J., and Oh, J. H.: Mechanical Design of Humanoid Robot Platform KHR-3 (KAIST Humanoid Robot-3: HUBO). In: Proc. of IEEE/RAS Int. Conf. on Humanoid Robots (HUMANOIDS), Tsukuba, 321-326, 2005.
Pfeiffer, F.: Technological Aspects of Walking, in: Walking: Biological and Technological Aspects, edited by: Pfeiffer, F. and Zielińska, T., Springer, Wien, 119-154, 2004.

Rose, J. and Gamble, J. G.: Human walking (3rd Edn.), Lippincott Williams \& Wilkins, Philadelphia, 2006.

Sakagami, Y., Watanabe, R., Aoyama, C., Matsunaga, S., Higaki, N., and Fujimura, K.: The Intelligent ASIMO: System Overview and Integration, in: Proc. of IEEE/RSJ Int. Conf. on Intelligent Robots and Systems (IROS), Lausanne, 3, 2478-2483, 2002.

Siciliano, B. and Khatib O.: Handbook of robotics, Part G, Legged Robots, Springer, Heidelberg, 361-390, 2008.

Sugahara, Y., Sugahara, Y., Endo, T., Lim, H. O., and Takanishi, A.: Design of a Battery-powered Multi-purpose Bipedal Locomotor with Parallel Mechanism, n: Proc. Int. Conf. on Intelligent Robots and Systems (IROS), Lausanne, 3, 2658-2663, 2002.

Tsai, L. W.: Robot Analysis: The Mechanics of Serial and Parallel Manipulators, John Wiley \& Sons, New York, 1999.

Tsai, L. W. and Joshi, S.A.: Kinematics and optimization of a spatial3-UPU parallel manipulator, ASME J. Mechan. Design, 122, 439-446, 2000.

Tsai, L. W. and Joshi, S.: Comparison Study of Architectures of Four 3 Degree-Of-Freedom Translational Parallel Manipulators, n: Proc. of the 2001 IEEE Int. Conf. on Robotics and Automation (ICRA), Seoul, 1283-1288, 2001.

Wang, H. B., Qi, Z. Y., Hu, Z. W., and Huang Z.: Application of Parallel Leg Mechanisms in Quadruped/Biped Reconfigurable Walking Robot, J. Mechan. Eng., 45, 24-30, 2009.

Wang, M. F. and Ceccarelli, M.: Design and Simulation for Kinematic Characteristics of a Tripod Mechanism for Biped Locomotors, in: Proc. of Intl. Workshop on Robotics in Alpe-AdriaDanube Region (RAAD), Portorož, 124-131, 2013.

Zhang, C. J. and Li, Y. W.: A New Walking Robot Based on 3-RPC Parallel Mechanism, Chinese J. Mechan. Eng., 47, 25-30, 2011 (in Chinese).

Zielinska, T.: Motion Synthesis, in: Walking: Biological and Technological Aspects, edited by: Pfeiffer, F. and Zielińska, T., Springer, Wien, 155-191, 2004. 